\title{
UK cancer survival statistics: rebuttal to editorial saying cancer survival statistics are misleading
}

In this rebuttal letter by Michel P Coleman and colleagues (BMJ 2011;343:d4214, doi:10.1136/bmj.d4214), the authors quote from Beral and Peto's original editorial (BMJ 2010;341:c4112, doi:10.1136/bmj.c4112). In the full text, online version of their letter, the italic emphasis that Coleman and colleagues had added to certain parts of this quotation was lost (because the whole quotation was published in italic). The quotation in their letter should show Coleman and colleagues' italic emphasis as follows: "If the first months or years of the illness are never traced, the earliest event registered may be some aspect of cancer recurrence. The date of this recurrence would then be taken as the date from which 'survival rates' are calculated. This makes short term survival look misleadingly worse in the UK than in countries such as Sweden ...." The print version is correct and can also be seen online (www.bmj.com/content/343/7814/ Letters.full.pdf).

Cite this as: BMJ 2011;343:d4522

๑ BMJ Publishing Group Ltd 2011 